\title{
MEMETIC ALGORITHM BASED ON HILL CLIMBING ALGORITHM FOR IC PARTITIONING
}

\author{
K. Jeya Prakash \\ Assistant Professor, ECE Department, \\ Kalasalingam Academy of Research and Education \\ (Deemed to be University). \\ Krishnankoil, (India). \\ E-mail: k.jeyaprakash@klu.ac.in ORCID: https://orcid.org/0000-0001-7493-1914 \\ P. Sivakumar \\ Professor, ECE Department, \\ Kalasalingam Academy of Research and Education \\ (Deemed to be University). \\ Krishnankoil, (India). \\ E-mail: siva@klu.ac.in ORCID: https://orcid.org/0000-0003-1328-8093
}

Recepción: 05/12/2019 Aceptación: 17/12/2019 Publicación: 23/03/2020

\section{Citación sugerida:}

Jeya Prakash, K., y Sivakumar, P. (2020). Mememtic algorithm based on hill climbing algorithm for IC partitioning. 3C Tecnología. Glosas de innovación aplicadas a la pyme. Edición Especial, Marzo 2020, 181-193. http://doi.org/10.17993/3ctecno.2020.specialissue4.181-193

\section{Suggested citation:}

Jeya Prakash, K., \& Sivakumar, P. (2020). Mememtic algorithm based on hill climbing algorithm for IC partitioning. 3C Tecnología. Glosas de innovación aplicadas a la pyme. Edición Especial, Marzo 2020, 181 193. http://doi.org/10.17993/3ctecno.2020.specialissue4.181-193 


\section{ABSTRACT}

To reduce the premature convergence of the optimization problem, the genetic algorithm with local search called "memetic algorithm" is introduced. The proposed memetic algorithm can partition a complex circuit design into a few sub-circuits. The aim of this paper is to reduce the interconnects between the partitioned blocks. The experimental results show that the method is effective for solving the given input and to find the minimum cut size. Applying memetic algorithm like Hill Climbing algorithm for 3D IC partitioning is the novelty in this work.

\section{KEYWORDS}

Memetic algorithm, Genetic algorithm, Gircuit partition, Cut size. 


\section{INTRODUCTION}

Very-large-scale integration (VLSI) is a process which integrates many transistors into a single chip called "Integrated Circuit". An electronic circuit requires many sub circuits like CPU, ROM, RAM and other glue logic. VLSI made it possible to include all of them into one chip. Designers depend on Computer Aided Design (CAD) tools to provide a higher level of idea to reduce the complexity of circuits.

The phrase related with the mission of automatically designing a circuit by means of CAD tools is known as Electronic Design Automation (EDA). In VLSI design, physical design is one of the steps in the standard design cycle which trails the circuit design as shown in Figure 1. At this step, circuit representation of the devices and interconnects of the design are changed into geometric representations of shapes which, at the point when produced in the relating layers of materials, will guarantee the essential functioning of the components. This geometric representation is called IC layout.

Circuit partitioning is a vital step which ensures the interactions between circuit blocks is minimal. The minimal inter-partition communication may lead to have a few numbers of wires between them. This in turn leads to small interconnect delay and low power.
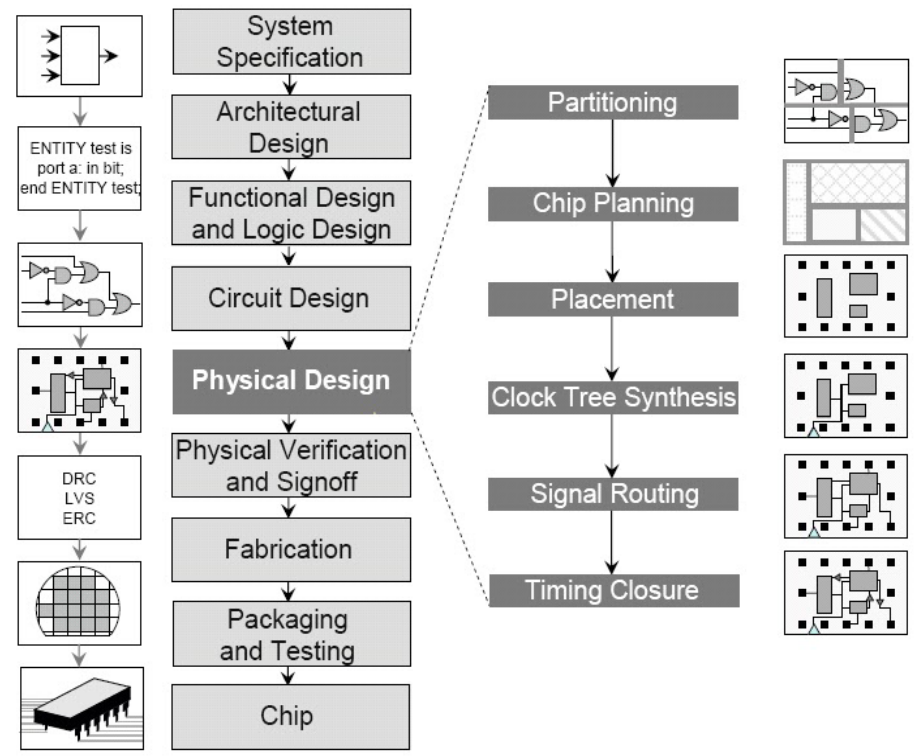

Figure 1. Design flow of VLSI. 
Hence, the main goal is partitioning a circuit into multiple blocks with an attempt to lessen the cut-size.

\section{PROBLEM FORMULATION}

In circuit partitioning problem, the logic representation of the circuit, modules and interconnection between modules are represented as geometric representation, vertices $(\mathrm{V})$ and edges $(\mathrm{E})$ of a graph $(\mathrm{G})$ respectively. The vertices and edges of $\mathrm{G}$ may be weighted to reveal module area or significance of an interconnection. The circuit partitioning has the following goals to make the IC compact:

Minimum Gut: Given $G=(V, E)$, partition $\mathrm{V}$ into disjoint subsets $\mathrm{X}$ and $\mathrm{Y}$ such that e $(\mathrm{X}$, $\mathrm{Y}$ ), the number of edges in $\{(\mathrm{x}, \mathrm{y}) \in \mathrm{E} \mid \mathrm{x} \in \mathrm{X}, \mathrm{y} \in \mathrm{Y}\}$, is minimized.

Minimum-Width Bisection: Given $G=(V, E)$, partition $\mathrm{V}$ into disjoint subsets $\mathrm{X}$ and $\mathrm{Y}$, with $|X|=|Y|$, such that $\mathrm{e}(\mathrm{X}, \mathrm{Y})$ is minimalized. Since this leads to equal number of modules in each partition, it is needed. The more general partitioning problem is when $\mathrm{k}$ disjoint subsets are formed.

Given two $n^{*} n$ matrix $X=\left(x_{i j}\right)$ and $Y=\left(y_{i j}\right)$, where usually $x_{i j}, y_{i j}>0$, and the objective is:

$$
\min \left(\pi \varepsilon S_{n}\right) \sum_{i=1}^{n} \sum_{j=1}^{n} x_{i j} y_{\pi(i) \pi(j)}
$$

where $\mathrm{Sn}$ is set of all probable permutation of $(1,2 \ldots \ldots \mathrm{n})$. Sometimes there is an accessory $n * n$ matrix $Z=\left(z_{i j}\right)$, then the equation becomes,

$$
\min \left(\pi \varepsilon \mathrm{S}_{\mathrm{n}}\right) \sum_{\mathrm{i}=1}^{\mathrm{n}} \sum_{\mathrm{j}=1}^{\mathrm{n}} \mathrm{X}_{\mathrm{ij}} \mathrm{y}_{\pi(\mathrm{i}) \pi(\mathrm{j})}+\sum_{\mathrm{i}=1}^{\mathrm{n}} \mathrm{z}_{\mathrm{i} \pi(\mathrm{j})}
$$

$\boldsymbol{x}_{i j}$ represents the flow from the module i to the module $\mathrm{j}$,

$\boldsymbol{y}_{i j}$ represents the distance from the location i to the location $\mathrm{j}$,

$z_{i j}$ represents the cost of the placing module $i$ to the location $j$. 


\section{MEMETIC ALGORITHM}

In evolutionary computation, the Memetic algorithms (MA) play a vital role in growing areas of research. The word MA is now broadly used as a synergy of any global search procedure with local enhancement procedures for problem search.

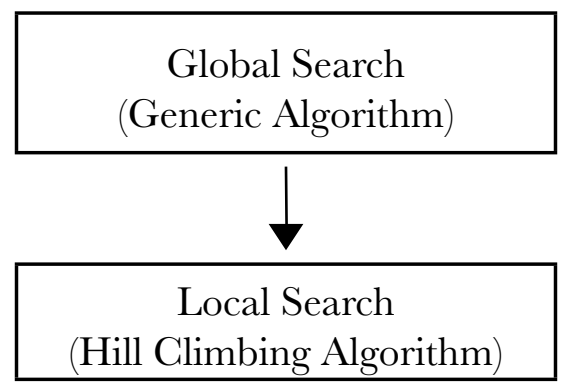

Figure 2. Memetic Algorithm.

The memetic algorithm, which is shown in Figure 2 is utilized to reduce the interconnections, i.e. min-cut problem of circuit partitioning based on a balanced limitation.

\subsection{GENETIC ALGORITHM}

A global exploration procedure to solve optimization problem, which evolves toward better solution, known as Genetic algorithm, is shown in Figure 3.

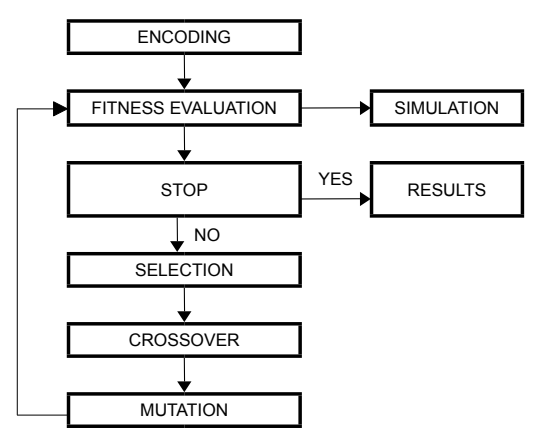

Figure 3. Genetic Algorithm- Flow Chart.

Encoding: The parameter like wire-length are represented as fixed length binary strings.

Initialization: Refers to generation of population of 'n' chromosomes randomly. Here, the population is the tentative solution for the problem. The population is here initialized by Roulette wheel Selection or Tournament methods. 
Fitness Evaluation: Evaluate the fitness $\mathrm{f}(\mathrm{x})$ of each chromosome $\mathrm{x}$ in the populace

Selection: In this process, the better fit two strings are selected as two parent chromosomes to create the offspring.

Grossover: Two parent qualities are mixed to get new children so that solution characteristics get changed. A probability is associated with this.

Mutation: With a mutation probability mutate new offspring to introduce new properties.

Termination: The process continues and replaces the existing solution until the termination condition is satisfied.

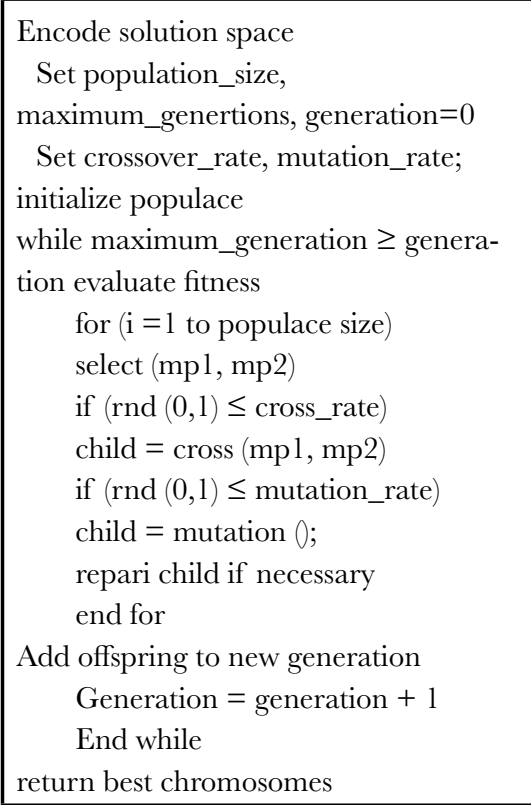

Figure 4. Pseudo code for Genetic Algorithm.

The algorithm takes specific paces, Initialization, Evaluation, Selection, Crossover, and Mutation. Every time, each person's fitness in the populace is evaluated. The fitness is typically the assessment of the target work in the issue being tackled. The best individual is preferred arbitrarily from the present populace and every individual's chromosomes and qualities are altered to make the fittest. The new populace is then used in the algorithm. The algorithm will end after predefined number of populaces are produced or achieved the optimal fitness function. 


\subsection{HILL CLIMBING ALGORITHM}

As stated in Lin and Zhu (2014), the GA is not fit for finding solutions which have closed to optimal solutions. Hence, usually GA is combined with local search algorithms like Hill climbing algorithm called Memetic Algorithms are used. In this paper we proposed a Memetic algorithm based on Genetic Algorithm and Hill climbing algorithm for circuit partitioning. Hill climbing algorithm is one of the algorithms to find the best state in optimization problems with less conditions than other techniques.

The genetic algorithm is not appropriate for fine-tuning the solution which are close to optimal. So, for fine tuning separate algorithm (local hill climbing algorithm) is used with genetic algorithm called Memetic. They have demonstrated that they are requests of greatness speedier than customary hereditary Algorithms for some issue areas. In a memetic algorithm, the population is initialized randomly or using a heuristic. Then, every individual makes nearby search to enhance its wellness. To frame another populace for the following group, higher quality solutions are chosen. The selection stage is similar stage. With two individuals selected, their chromosomes are joined to produce new individuals.

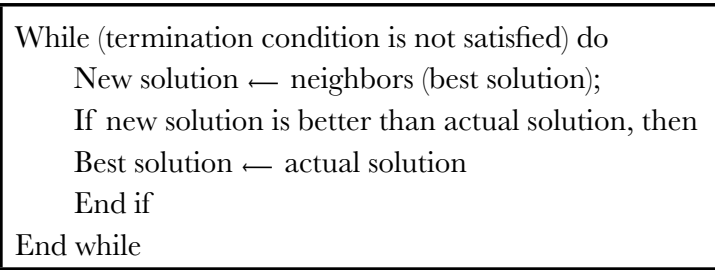

Figure 5. Pseudo Code for Hill climbing search algorithm.

The later are boosted utilizing a neighbourhood seek method. The role is to trace the local best more proficiently than the genetic algorithm. The hill climbing search algorithm proposed as a local search procedure shown in Figure 5. It is just a loop that ceaselessly goes toward expanding quality.

\section{RESULTS}

The parameter settings of iteration are varied, and the cut size is calculated. The best cost for various iterations up to 20 iterations as example, is taken in partitioning ami33 is shown in figures below: 


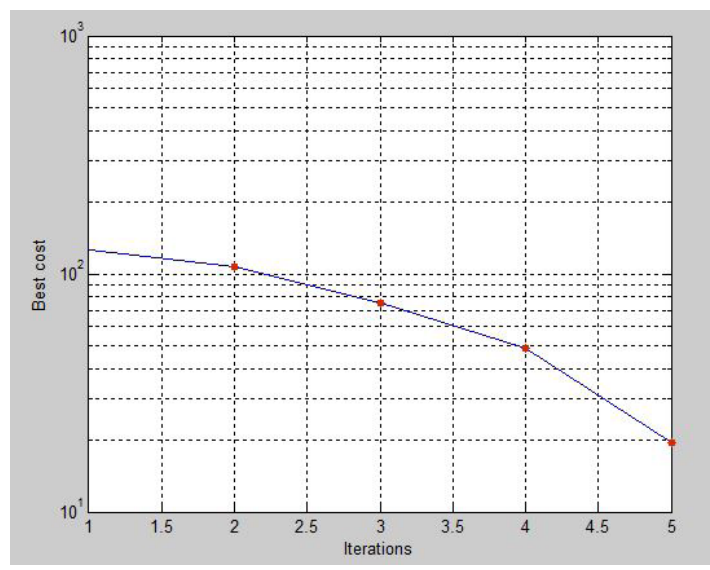

Figure 6. Iterations Vs. Best cost (for Maxlt=5).

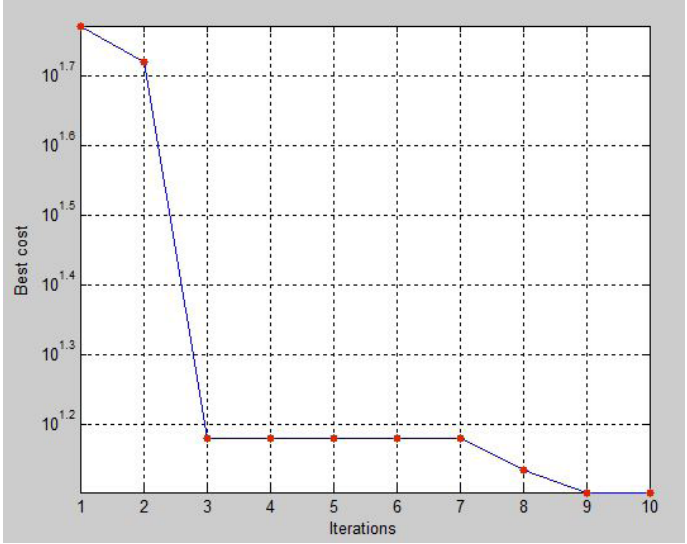

Figure 7. Iterations Vs. Best cost (for Maxlt=10).

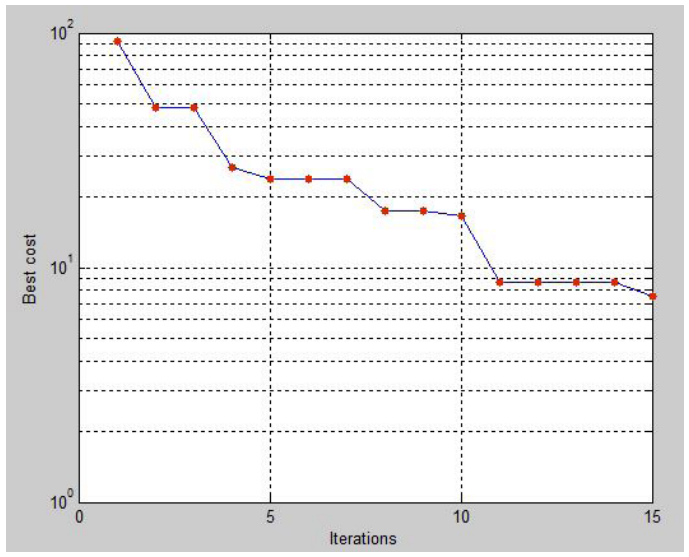

Figure 8. Iterations Vs. Best cost (for Maxlt=15). 


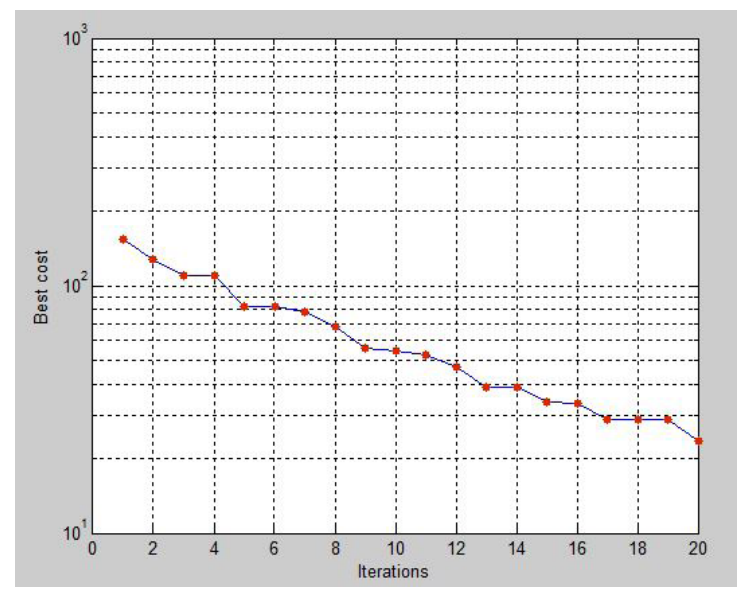

Figure 9. Iterations Vs. Best cost (for Maxlt=20).

The tabulation for memetic algorithm of min cut, max cut and average cut is shown in the Table 1.

Table 1. Min cut, max cut and average cut of partitioning.

\begin{tabular}{|c|c|c|c|c|}
\hline Iterations & Min cut & Max cut & Average cut & Memetic Mean (*106) \\
\hline 5 & 32.06 & 576.02 & 29.03 & 5.26 \\
\hline 10 & 32.38 & 544.03 & 27.2 & 5.26 \\
\hline 15 & 33.5 & 544.08 & 28.29 & 5.44 \\
\hline 20 & 32.40 & 320.00 & 19.20 & 5.44 \\
\hline
\end{tabular}

The results clearly show that the proposed work results in one of the best ways to partition a 3D IC.

\section{CONCLUSION}

The combination of genetic algorithm with local hill climbing algorithm forms a memetic algorithm which is proposed to circuit partitioning yields a major development in result quality. The experimental result shows that the algorithm provides good and consistent result. This result shows the flexibility of the memetic methodology in solving the problem of VLSI circuit netlist partitioning. 


\section{ACKNOWLEDGEMENT}

Authors want to express their gratitude to ECE Department at Kalasalingam Academy of Research and Education for allowing them to utilize the computing facilities in DST-FIST sponsored VLSI Research Laboratory. 


\section{REFERENCES}

Ababei, G., Selvakkumaran, N., Bazargan, K., \& Karypis, G. (2002). Multi-objective circuit partitioning for cutsize and path-based delay minimization. Proceedings of the 2002 IEEE/ACM International Conference on Computer-Aided Design - ICCAD 02, 181-185. https://doi.org/10.1145/774572.774599

Alpert, C.J. (1998). The ISPD98 circuit benchmark suite. Proceedings of the 1998 International Symposium on Physical Design - ISPD 98. https://doi.org/10.1145/274535.274546

Areibi, S., \& Yang, Z. (2004). Effective Memetic Algorithms for VLSI Design = Genetic Algorithms Local Search Multi-Level Clustering. Evolutionary Computation, 12(3), 327353. https://doi.org/10.1162/1063656041774947

Du, H. Q., \& Qi, J. B. (2010). Application of a Hybrid Algorithm Based on Genetic Algorithm and Hill-Climbing Algorithm to Tool Path Optimization in CNG Machining. Advanced Materials Research, 102-104, 681-685. https://doi.org/10.4028/ www.scientific.net/amr.102-104.681

Gill, S. S., Chandel, R., \& Chandel, A. (2010). Genetic Algorithm Based Approach to Circuit Partitioning. International Fournal of Computer and Electrical Engineering, 196-202. https://doi.org/10.7763/ijcee.2010.v2.136

Lin, G., \& Zhu, W. (2014). An Efficient Memetic Algorithm for theMax-Bisection Problem. IEEE Transactions on Computers, 63(6), 1365-1376. https://doi.org/10.1109/tc.2013.7

Marichelvam, M. K., Prabaharan, T., \& Yang, X. S. (2014). A Discrete Firefly Algorithm for the Multi-Objective Hybrid Flowshop Scheduling Problems. IEEE Transactions on Evolutionary Computation, 18(2), 301-305. https://doi.org/10.1109/ tevc.2013.2240304

Nagarajan, K. (2018). A Predictive Hill Climbing Algorithm for Real Valued multiVariable Optimization Problem like PID Tuning. International fournal of Machine Learning and Computing, 8(1), 14-19. https://doi.org/10.18178/ijmlc.2018.8.1.656 
Roy, S., \& Sarma, S. S. (2012). Improvement of the quality of VLSI circuit partitioning problem using Genetic algorithm. Fournal of Global Research in Computer Science, 3(12), $18-22$.

Sharma, P. K., \& Kaur, M. (2014). A Discrete FireFly Algorithm for VLSI Circuit Partitioning. 2014 International Conference on Electronics and Communication Systems (ICECS). https://doi.org/10.1109/ecs.2014.6892764

Subbaraj, P., Sivasundari, K., \& Kumar, P. (2007). An effective memetic algorithm for VLSI partitioning problem. IET-UK International Conference on Information and Communication Technology in Electrical Sciences (ICTES 2007), 667-670. https://doi. org/10.1049/ic:20070696 
